\title{
Some questions of epidemiology, statistics, and pandemic forecast COVID-19.
}

\author{
Shamil Timerbulatov ${ }^{1}$, Vil Timerbulatov ${ }^{1}$, and Makhmud Timerbulatov ${ }^{1}$ \\ ${ }^{1}$ Bashkir State Medical University
}

September 11, 2020

\begin{abstract}
The article analyzes the indicators of prevalence, morbidity, lethality and mortality in the new coronavirus disease - COVID-19, based on this specialized literature and official information from the state mass media. The importance of correct application of the principles of medical statistics for the analysis of these indicators in decision-making on the introduction of restrictive measures, monitoring the epidemic process, evaluating the effectiveness of these measures and the activities of medical organizations is shown. Individual pandemic forecast models are considered.
\end{abstract}

Some questions of epidemiology, statistics, and pandemic forecast

COVID-19.

Prof. Vil M. Timerbulatov, MD, Shamil V. Timerbulatov, MD, Prof. Mahmud V. Timerbulatov, MD.

Bashkir State Medical University, str. Lenin, 3, Ufa, Russian Federation. 450008.

Professor Vil Mamilovich Timerbulatov - head of surgery department in Bashkir State Medical University, Ufa, Russian Federation; head of Surgery Emergency Clinic, Ufa, Russian Federation; https://orcid.org/00000002-1696-3146;

Professor Shamil Vilevich Timerbulatov - PhD, surgery department in Bashkir State Medical University, Ufa, Russian Federation; deputy head of Clinic for Infection Diseases, Ufa, Russian Federation; https://orcid.org/0000-0002-4832-6363; e-mail:timersh@yandex.ru; phone: +79273073333;

Professor Makhmud Vilevich Timerbulatov - head of surgery department in Bashkir State Medical University, Ufa, Russian Federation; head of Surgery Emergency Clinic, Ufa, Russian Federation; https://orcid.org/00000002-6664-1308.

Number of words 5634 .

The article analyzes the indicators of prevalence, morbidity, lethality and mortality in the new coronavirus disease - COVID-19, based on this specialized literature and official information from the state mass media. The importance of correct application of the principles of medical statistics for the analysis of these indicators in decision-making on the introduction of restrictive measures, monitoring the epidemic process, evaluating the effectiveness of these measures and the activities of medical organizations is shown. Individual pandemic forecast models are considered.

Since the World Health Organization's announcement on 11 March 2020 of a new coronavirus disease pandemic, COVID-19, many reports of illness, especially deaths, from the media, social networks, have triggered a stormy, mixed public reaction and often fueled fear and depression in people. 
In statistical processing of medical articles, packages of statistical programs are used, based on probability theory and mathematical statistics [1], i.e., in fact, the techniques of "medical statistics" are used. The complexity of statistical analysis of medical data is caused by a number of objective and subjective factors, such as specific standards in research design, the presence of legal, economic, ethical restrictions, the great complexity of the studied phenomena, the presence of "leads" and confusing factors, the most frequently solved problems do not completely coincide with those in other subject areas.

In the first month of the pandemic, official information from WHO and all countries reported numbers of people infected and dead (in absolute figures), with shocking effects on the population, although these figures did not reflect the status, course or trends of the epidemic. Such uncertainty in the assessment of the situation, especially ignorance of trends in the spread of COVID-19, could not contribute to the adoption of correct, justified, especially preventive decisions by the authorities, public health leaders, at least in the near future.

As of May 26, 2020 COVID-19 in the world 5,603,427 people got sick, 350,041 died, in the Russian Federation 362,342 and 3,807 respectively. The mortality rate for this infection in the world is $6.5 \%$, the share of the infected population is $0.06445 \%$, in Russia it is $0.96 \%$ and $0.22 \%$ respectively.

A pandemic occurs when 1 to $5 \%$ of the population is affected, but given the characteristics of the COVID-19 coronavirus, WHO has declared a pandemic with lower infection rates.

Up to $60 \%$ of those tested in Russia have the coronavirus without symptoms. The results of antibody testing of the population in Santa Clara County, California, showed that the morbidity was 50-85 times higher than the official one and, accordingly, the mortality rate was also 50-85 times lower and was $0.04-0.07 \%$ instead of $4 \%$.

It is important to clearly distinguish between lethality (\% of all deaths) and mortality, which is calculated on 1,000 or more often on 100 thousand population.

Every year 1.2 million people die from pneumonia alone, 350,041 died from coronavirus between the end of December 2019 and 26 May 2020, but the difference between dying with coronavirus and dying from coronavirus must also be considered. The vast majority of those who died suffered from cancer, chronic lung disease, obesity, diabetes, and cardiovascular disease, and the virus was the last straw during their illnesses.

According to Rospotrebnadzor (Russian Federal Service for Supervision of Consumer Rights Protection and Human Health), following the results of the influenza and acute respiratory infections epidemic in 2016-2017, the daily number of cases in Russia was 120 thousand people, which is significantly higher than COVID-19 (from 5 thousand to 10 thousand per day). Therefore, rather, the peak of the epidemic in Russia was passed 2 months ago, when there was no testing due to the total hospitalization of all infected people in hospitals, the latter have become real centers of the hospital epidemic (in $55 \%$ of centers in medical organizations), the expansion of indications was influenced by the increase in the cost of payment for medical insurance up to 200 thousand rubles per case. With the further mass conversion of medical organizations into infectious disease hospitals, the latter are loaded only by $30-35 \%$.

On 15 January 2019 WHO announced a list of 10 major threats to human health in 2019, including air pollution and related climate change, Ebola, the global influenza pandemic, HIV and increased resistance of bacteria to antibiotics.

Every year, air pollution leads to the premature death of 7 million people.

Non-communicable diseases such as diabetes, cancer, and cardiovascular diseases are the second most dangerous, with 4 million people dying from them every year ( $7 \%$ of all deaths).

As of 31 January 2020, 213 people worldwide had died infected with COVID-19. During this period, 684,497 people died of cancer, 633,508 children under 5 years old, 140,112 suicides, 81,757 of malaria, 70,189 of contaminated water, 40,452 of influenza, 25,763 during childbirth. 
As of March 11, 2020, the total number of COVID-19 infections in the world exceeded 118 thousand in 114 countries, according to WHO, 4,291 people died. Most of the infected were found in Italy $(>10,000)$, Iran (9,000 cases, of which almost 1,000 died), South Korea (7,700, 242 died), and the Russian Federation had 20 infected, so at the beginning of the pandemic the global mortality rate from COVID-19 was 3.63\%, in Iran - $11.1 \%$, South Korea - 3.14\%, in Russia - 0\%.

Subclinical or mild forms of coronavirus disease were observed in $80.9 \%$ of patients, but they had the potential for virus spread [2] and had the same viral load as patients with symptoms of SARS-CoV-2 [3].

According to WHO, the incubation period is 5.2 days with a range of 1-14 days [4].

The average time for patients to see a doctor from the onset of symptoms is 5.8 days, and 12.5 days before hospitalization [5].

The stages of symptomatic disease were classified on the basis of non-contrast CT results and divided into early (0-4 days), progressive (5-8 days), peak (9-13 days) and adsorption ( $>14$ days) stages [6].

Subpleural opacities (GGOs) located in the lower lobes of the lung are detected at an early stage of the disease. Bilateral spread of the infection process and diffuse subpleural turbidity are detected in the progression stage. At the peak stage, the presence of dense consolidation, residual parenchymatous strips are determined. The adsorption stage can last more than 26 days, with a more controlled process, gradual resolution, and signs of recovery [6].

In a prospective cohort study of 1,150 COVID-19 patients hospitalized in New York City, it was shown that more than $\frac{3}{4}$ of the severely ill patients needed a ventilator and almost $1 / 3$ needed hemodialysis [7]. Of the patients in critical condition, $39 \%$ died, and $41 \%$ received invasive mechanical ventilation.

According to the National Health Service of England, Wales and Northern Ireland (11,292 patients needed intensive care), $50 \%$ of patients who received a ventilator died within 40 days after starting intensive care [8].

In the United States, at the beginning of the pandemic, there were 95,000 intensive care and resuscitation beds (28.7 beds per 100,000 population). In Russia there is no exact data, their number, according to different sources, varies from 12 thousand to 35 thousand (8.57-35.0 per 100,000 population).

The number of COVID-19 intensive care beds also varies greatly in different countries from 22.9 beds 100,000 population in Germany, 28.9 - Austria, 16.3 - France, 12.9 - Canada, 10.5 - UK, 9.7 - Spain. 8.6 - Italy, 7.8 - Denmark, 5.0 - Ireland.

In 2016, there were 197 million pneumonia cases worldwide and 1,200,000 died.

In 2017, according to the Federal State Statistics Service, over 671 thousand people suffered from pneumonia in Russia, and 26 thousand died. 26,088 died in 2017, 25,642 people died in 2018.

In 2019, 1.8 million people died from various causes in Russia, 1.67 million died directly from diseases, and 130 thousand died from external causes.

In Russia, every year respiratory diseases (influenza, acute respiratory infections, pneumonia) are carried by [?]50 million people.

According to WHO, there are [?]1 billion influenza cases worldwide every year, including 3-5 million severe cases and from 300 to 650 thousand deaths.

Table 1.

Rates of morbidity and mortality of new coronavirus infection COVID-19 in different countries (as of 30.05.2020) 


\begin{tabular}{llll}
\hline Country & $\begin{array}{l}\text { Density of population per } \\
\text { square kilometer }\end{array}$ & $\begin{array}{l}\text { Incidence per 100,000 } \\
\text { population }\end{array}$ & Lethality (in \%) \\
\hline Russia & 9 & 283.2 & 1.14 \\
USA & 32 & 540.3 & 5.86 \\
Italy & 193 & 387.0 & 14.3 \\
Spain & 92 & 620.8 & 10.06 \\
France & 118 & 281.7 & 15.20 \\
Great Britain & 255 & 484.2 & 14.07 \\
Germany & 230 & 220.48 & 4.69 \\
Brazil & 23.6 & 223.0 & 5.96 \\
Turkey & 100 & 195.3 & 2.76 \\
China & 650 & 6.0 & 5.5 \\
In the world & 12 & 85.22 & 6.09 \\
Norway & 13 & 157.09 & 2.8 \\
Sweden & 20 & 1060.0 & 11.84 \\
\hline
\end{tabular}

It can be noted from the presented data (Table 1) that there is no unambiguous dependence of morbidity and detail indicator on population density.

Experience with the COVID-19 pandemic since 11 March 2020 shows that countries that introduced severe restrictive measures early in the epidemic have significantly reduced or stabilized their infection (morbidity) rates. Such countries include China, Russia, Germany, Norway (morbidity per 100,000 population $<300$ people), and the countries that followed a "liberal approach" to restrictive measures had morbidity rates above 300/100,000 population (Italy, Spain, United Kingdom). The absence of practically restrictive measures has led to a significant increase in the incidence of diseases, as happened in Sweden ( $>1,000 / 100,000$ population).

There are other reasons for fluctuations in the incidence rate. These include the loyalty of the population to the authorities, the implementation by the vast majority of the population of measures aimed at preventing the spread of infection. The need for mandatory implementation of recommendations by citizens should be accompanied by informing the population about the causes of the spread of infection and possible consequences of the disease. It is important to have truthful information based on the opinions of medical scientists, the maximum exclusion of pseudo-scientific, previously false information about the infection. The third aspect of this problem is the development of a control system and analysis of the implementation of restrictive measures. The state solves this issue in different ways and there can be no right recipes for everyone.

In addition, long-term restrictive measures of isolation of citizens cause fatigue, which leads to disregard for social distances, wearing masks.

Rating of countries on the number of deaths from coronavirus infection as of 18.04.2020 led the U.S., Britain, Italy, France, Spain, but in calculating mortality - the situation is different. Belgium (16.37\%), France (16.64\%), Great Britain (14.17\%), Italy (14.15\%), Hungary (13.07\%), Netherlands (12.90\%), Sweden (12.21\%), Spain (11.95\%), Mexico (10.52\%), USA (5.1\%), Germany (3.07\%), Brazil (6.3\%), Turkey (2.16\%), China $(5.46 \%)$, Norway $(1.31 \%)$, Russia $(0.94 \%)$.

Table 2.

Comparative lethality rates in some countries for 18.04.2020 and 30.05.2020.

\begin{tabular}{lll}
\hline Country & Mortality rates (in \%) & Mortality rates (in \%) \\
\hline \multirow{3}{*}{ Russia } & On 18.04.2020 & On 30.05.2020 \\
& 0.94 & 1.14
\end{tabular}




\begin{tabular}{lll}
\hline Country & Mortality rates (in \%) & Mortality rates (in \%) \\
\hline USA & 5.1 & 5.86 \\
Italy & 14.15 & 14.3 \\
Spain & 11.95 & 10.06 \\
France & 15.64 & 15.20 \\
Great Britain & 14.17 & 14.07 \\
Germany & 3.07 & 4.69 \\
Brazil & 6.3 & 5.96 \\
Turkey & 2.16 & 2.76 \\
China & 5.46 & 5.5 \\
Norway & 1.31 & 2.80 \\
Sweden & 12.21 & 11.84 \\
\hline
\end{tabular}

In most countries, there has been virtually no marked change in mortality rates, some increase in mortality in the USA (from 5.1 to $5.86 \%$ ), Germany (from 3.07 to $4.69 \%$ ), Norway (from 1.31 to $2.8 \%$ ), was statistically unreliable (OR: 0.833 (95\% CI, 0.262-2.642), $\mathrm{p}=0.756 ; 0.600$ (96\% CI, 0.147-2.433), $\mathrm{p}=0.475$; and $0.33(95 \%$ CI, 0.35-3.150), $\mathrm{p}=0.337$ ) respectively.

The persistence of a stable level of lethality in coronavirus disease seems to be due to the difficulties of etiopathogenetic treatment of this viral infection. The existing methods of medical treatment are extremely variable, the drugs used were mostly recommended by the principle of "repositioning" drugs and imply the search for drugs for the treatment of a new disease among the substances already studied in clinical trials or used in clinical practice [9]. Almost to this day, there are no drugs whose effectiveness has been proven by the results of randomized controlled trials and meta-analyses. An example is the recent WHO recommendations not to use chloroquine/hydroxychloroquine that are not based on strong evidence in the treatment of coronavirus disease. 96,032 patients with COVID-19 from 20.12.2019 to 14.04.2020 were analyzed for the effectiveness of treatment in 671 hospitals on 6 continents [10].

10,698 (11.1\%) of them died in hospitals, 14,888 patients formed 4 groups: within 48 hours after diagnosis, they began receiving chloroquine $(1,868)$, chloroquine with macrolides $(3,783)$, hydroxychloroquine $(3,016)$ and hydroxychloroquine with macrolides (6,221 patients). 81,144 patients did not receive treatment for any of the schemes made up the control group. In the control group, the mortality rate was $9.3 \%$, in the group receiving hydroxychloroquine $-18 \%$, hydroxychloroquine with macrolides $-23.8 \%$, chloroquine $-16.4 \%$, and chloroquine with macrolides $-22.2 \%$.

Scientists from South Korea have screened about 3,000 approved drugs and found substances potentially effective against SARS-CoV-2 and considered niclosamide and cyclosonide the most promising [9]. The genomes of SARS-CoV and SARS-CoV-2 viruses coincide by $79.5 \%$, and therefore the authors suggested that drugs that suppress SARS-CoV may also be effective against SARS-CoV-2 [57].

Out of 3,000 molecules, 35 were identified that inhibit SARS-CoV-2. In the course of the experiment Vero cell culture (normal kidney cells of African green monkey) was treated with the drug, and then infected with SARS-CoV-2. 24 hours after the infection the immunofluorescent cell analysis was carried out with antibody that recognized SARS-CoV-2 nucleo-capsid protein - it was identified as the most significant 2 medications. Niklozamide, an antihelminthic drug used in tapeworm infestation, was very active against SARS-CoV-2 (IC50 semi-maximum inhibition concentration - $0.28 \mu \mathrm{M}$ ) and was effective against a wide range of viruses, including SARS-CoV and MERS-CoV. Ciclesonide, an inhaled corticosteroid, is used to treat bronchial asthma and allergic rhinitis, although its activity was significantly lower against SARS-CoV-2 $(\mathrm{IC} 50-4.33 \mu \mathrm{M})$.

Possible reasons for the lack of reduction in mortality can be a gradual reduction in the resources of medical organizations, health care in general, the growing shortage of medical personnel due to their morbidity, mortality, physical, moral and psychological fatigue, lack of proper material and moral support. 
In April, the unemployment rate in Sweden reached 7.9\%, excluding seasonal factors - 8.2\%. Bloomberg analyst Johanna Jeneson forecasts that unemployment will rise to $17 \%$. Thus, the Swedish economy, which refused to impose a strict quarantine, did not help.

Analysts of the Financial Times, comparing the mortality during the pandemic in 13 countries in MarchApril 2020 with the average for the same period in 2015-2019, noted an increase of $49 \%$ in 2020: in France $34 \%$, Italy $90 \%$, the Netherlands $40 \%$, Spain $51 \%$, Belgium $60 \%$, Sweden only $18 \%$.

But according to countrymeters.info, in January-March 1,580 people per day died, in 2019 - 1,586 people per day. In USA in the first three months of 2020, 4,477 people died per day, and in 2019 - 7,458 people died per day. Spain - 1,098 and 1,105, Germany - 2,400 and 2,410, Russia - 5,538 people per day (including from COVID-19 - 450 people) in 2020 and 5,563 people per day in 2019.

The infection rate in the Russian Federation in the middle of March was at the level of 3, 15 May - 0.9, for a full economic life 0.5 is required. As of May 15, 2020 in 28 regions the coefficient was $<1$, in 9 - about 3.

Hospitalized on May 20, 2020 in Russia - 109 thousand people, 2,500 - in the ICU. By severity: heavy $4.2 \%$, medium - $34.9 \%$, light $-60.9 \%$. Of the specialized 165,290 beds, 110 thousand (66\%) are used, $92 \%$ of the beds with ventilators are not occupied. Thus, $2.29 \%$ of patients with COVID-19 were hospitalized for intensive care and resuscitation. On the one hand, the high percentage of unused, especially expensive beds is a serious, additional burden on health care, which certainly contributes to growth, "accumulation" of patients waiting for planned, especially surgical treatment. But on the other hand, the complexity and unpredictability of the epidemiological process, the emergence of new outbreaks, justifies the over-deployment of infectious beds, at least until the infection rate $<1.0$, after the "plateau" of morbidity has been completed and the decline began.

By 25 May 2020, 3,807 people had died from COVID-19 in Russia, which would have been about $14.64 \%$ of all deaths in 2019 in Russia due to pneumonia $(26,000)$.

In 2019, the total mortality rate in the Russian Federation from all diseases was $1.2 \%$, and from coronavirus during the pandemic period was $0.98-1.05 \%$. Thus, in the structure of total lethality, deaths from coronavirus may be $0.21 \%$ (3,807 deaths from COVID-19 as of 26.05.2020 for a total of 1,800,000 deaths in 2019).

Concomitant diseases and infections lead to an increase in the severity of respiratory viral infections and mortality [11]. Most deaths during the 1918 influenza epidemic were caused by subsequent bacterial infection, especially Streptococcus pneumonia [11].

Poor outcomes of the $2009 \mathrm{H} 1 \mathrm{~N} 1 \mathrm{flu}$ pandemic were also associated with subsequent bacterial infections [12].

In the current COVID-19 pandemic, $50 \%$ of patients who died had secondary bacterial infections [13], with both bacterial and fungal infections reported [14].

Patients with COVID-19 are on an invasive ventilator for a long time (on average, 9.1 days), which increases the likelihood of developing hospital- and ventilator-associated infections.

It is important to prevent the second wave of the epidemic, which is predicted by many virologists in autumn-winter 2020 .

In Berlin, scientists suggest tightening the quarantine at 30 newly infected per week, then the extreme color of the epidemiological "traffic light" turns on, at 20 per 100,000 - warning yellow. The second factor - the coefficient - is the so-called coronovirus reproduction index, i.e. the number of people infected by one infected person. Only when this coefficient is $<1.0$ can the epidemic be kept under control. If for 3 days in a row 1 infected person will infect 1.1 healthy - the color of yellow is turned on, at a factor of 1.2 - red "traffic light", the third criterion - the number of beds for heavy patients with COVID-19 in the intensive care and intensive care units in the clinics. In the "quiet period" $9 \%$ of the beds are occupied, if this indicator reaches $15 \%$ yellow will light up, at $25 \%$ - red color of the epidemiological "traffic light" (http://p.dw.com/p/3cAza). 
With two yellow signals at 3 "traffic lights", the Berlin (Senate) management begins a thorough analysis of the situation, and with two red signals, it decides whether to introduce quarantine restrictions again, if yes, which ones.

Obviously, apart from these figures, it is important to monitor the dynamics of the epidemiological process.

German scientists have summed up the first results of the fight against COVID-19 in the country (DWBreaking World News, 20.05.2020) and among the key factors to contain the coronavirus epidemic in Germany, they call the decisive actions of the authorities, on the one hand, and the loyalty of the population to the decisions of the authorities, on the other. Now, after the easing of restrictive measures, in places of mass appearance of people, such measures as thermometry in front of the entrance to shops, mandatory hand disinfection, issuing masks to all visitors and inside the building, social distance is maintained.

According to German scientists (prof. Karl Gustav Karus University hospital Michael Albrecht) the new coronavirus has nothing to do with ARVI or flu. COVID-19 in the worst case affects not only the lungs, but also other internal organs, causes severe immunological reactions in them, affects the central nervous system. Scientists believe that the widely discussed problem of lack of ventilators is just one aspect of the treatment of patients with COVID-19. Thanks to the cancellation of public events and the prohibition of human contact, the spread of infection in Germany was stopped ( $h t t p: / / p . d w . c o m / p / 3 c A z a)$.

Similar experiences in the fight against the pandemic in Austria, the Czech Republic, China, Russia have also shown the effectiveness of such restrictive measures (morbidity, mortality, etc.).

According to various virologists from England and Germany, the pandemic may last for another one to two years. It is believed that COVID-19, on the one hand, will not disappear, and on the other, will not become an obstacle to the normalization of life, which will occur due to vaccination or the acquisition of immunity by the population naturally [7].

According to computer simulations of the pandemic from Johns Hopkins University, which were developed under the condition that antiepidemic measures were maintained or introduced, quarantine measures could be withdrawn in most countries no earlier than August-September 2020.

The emergence of the experimental vaccine and its application to the vaccination of medical personnel is predicted in November 2020, and mass vaccination in spring 2021 [8].

One of the most common models for predicting the development of epidemics is SIR, which was developed in the late 1920s (an acronym formed from the first letters of the words "susceptible", "infected" and "recovered") [15].

The essence of the SIR model is that the entire population is divided into the susceptible - those who may be infected, those who are actually infected and recovered.

Several variations of the models have been developed to date, including SEIR, where E means exposed, i.e. infected with the virus during the incubation period (contact). These people have already been infected, but the symptoms of the disease are not yet apparent. According to the SEIR model, susceptible people first become infected, then the incubation period lasts for some time, after which they develop symptoms and eventually recover or die. In the case of COVID-19, infected people can infect others before symptoms appear, which makes the virus particularly contagious (the incubation period of the coronavirus is on average 5-6 days) [16].

The infectivity (pathogenicity) of the virus is measured using the index (coefficient) of basic reproduction $\mathrm{RO}$, if it is equal to 1.0, then each person infects another person, and that person then passes the virus to another person, and so on. 


\section{How the infection will spread in different reproduction indices $(\mathbf{R})$}

$R$ - the number of people who infect the diseased

The number of sick, thousands of people

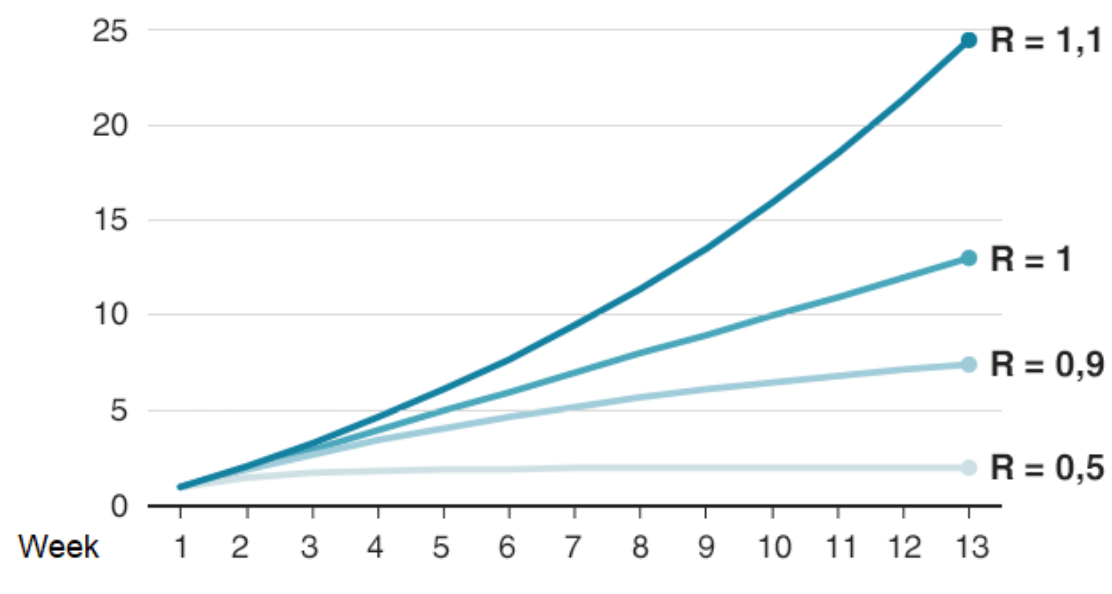

BBBC

Fig. 1 Distribution of infection with different indices of reproduction (R). (Source: Imperial College London). 


\section{How quarantine changed the Reproduction Index (R) in Britain}

$R$ - the number of people who infect the diseased

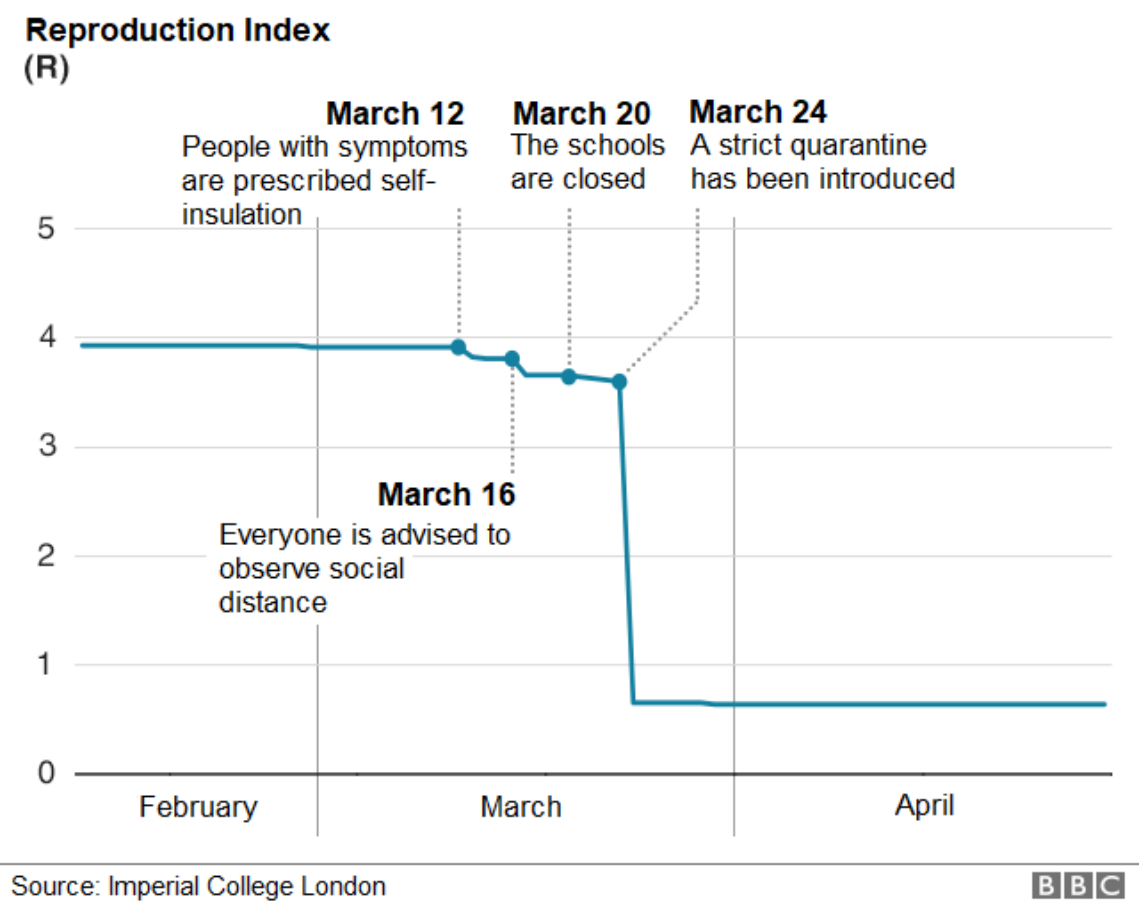

Fig. 2 Changes in the reproduction index $(\mathrm{R})$ in the UK under various restrictive measures. (Source: Imperial College London). 


\section{How would an epidemic develop in Russia without quarantine

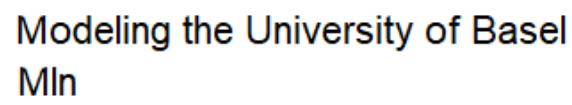

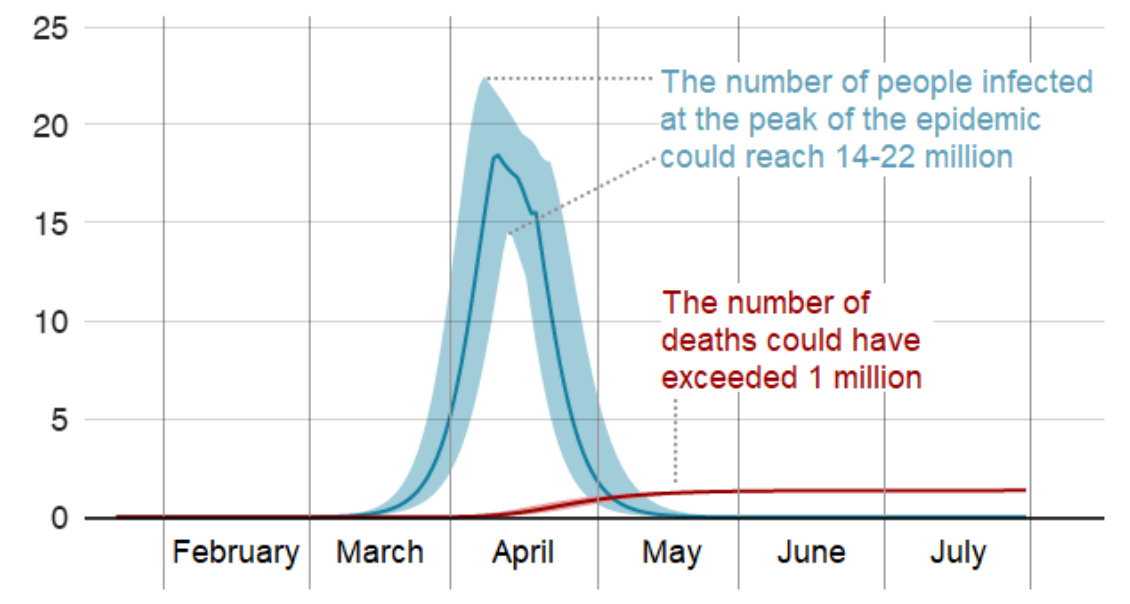

Source: covid19-scenarios.org

Fig. 3 Scenario of epidemic development in Russia without restrictive measures. (Source: Covid-19scenarios.org)

Increasing $\mathrm{RO}$ per unit significantly accelerates the process, if $\mathrm{RO}$ is 2, then the first infected person transmits the virus to two more people, then each of them transmits the virus to two more people and so on.

The index of the current COVID-19 coronavirus is about three. When the authorities restrict people's contacts, the reproduction index decreases. Now all countries are trying to lower the reproduction rate below 1, hoping that at a slow rate of infection, restrictive measures can be eased (Fig. 2).

Reducing the reproduction index does not yet mean defeating the virus, but a low infection rate can be controlled through more economical measures - wearing masks, mass testing, tracking contacts and timely isolation of people with symptoms.

In order to model the coronavirus distribution, the University of Basel, Switzerland, developed an interactive model based on SEIR, where data (number of inhabitants, age structure, number of confirmed cases, etc.) are loaded automatically and RO is assumed to be 2.64-3.23.

If no restrictive measures were taken in Russia, according to this model, 1.3-1.36 million people would have died by the beginning of July, and the number of infected people at the peak of the epidemic would have been 14-22 million (Fig. 3).

If Russia eases the restrictions so that the number of people's contacts will increase by $50 \%$, the number of new infections may increase to 300,000 by May 31, 2020, and if nothing is changed or the number of contacts reduced by another $50 \%$, the increase in infection will be almost invisible or not at all.

Based on mathematical modeling of COVID-19 epidemic development in Moscow, 5 scenarios of epidemic suppression and their possible consequences are presented [18]. The extended SEIR model [15], implemented as a public computer program, was used for modeling. 5 scenarios of the epidemic development are considered: 0 - zero scenario corresponds to the absence of protective measures, scenario A - a mild step to suppress the epidemic (closure of schools, universities, recommendations to elderly people not to leave home), scenario B and D - a complete knockdown, introduced in scenario B from the beginning of May, in scenario D - from 
the beginning of April 2020. In the O-variant, the number of deaths from COVID-19 in Moscow will exceed 100 thousand people, and the number of critical patients at the peak of the epidemic will exceed the capacity of the healthcare system by more than 10 times. Scenarios A and B do not radically reduce the number of deaths, and the number of critical patients at the peak of the epidemic will continue to far exceed the capacity of the health system. In addition, scenario B assumes that the epidemic will last for more than a year. Scenarios B and D can suppress the epidemic and significantly reduce the number of deaths (by 30 and 400 times, respectively). At the same time, as a result of these two scenarios, the population does not develop collective (group) immunity and the population will remain susceptible to repeated outbreaks of the epidemic.

Of the 50,000 Moscow citizens who voluntarily tested, antibodies to COVID-19 were found in 12.5-14\% who are developing or have developed immunity to the coronavirus. Rather, the examined patients suffered from the disease in an asymptomatic or mild form, without contacting medical professionals. Based on the results of these studies, it can be assumed that those who acquired immunity during the asymptomatic course of COVID-19 in Moscow may be from 1,586,000 to 1,776,000 people, and the total number of infected, including officially registered (applied for medical care) patients then is from 175,000 to 1,946,000 people. Consequently, the proportion of those identified in medical organizations is only $8.69-9.64 \%$ of the total number of infected people.

During the last month, the growth of COVID-19 cases has been gradually decreasing: for example, during April 15-19, 2020, this figure was 14-15\%, May 1-5, 2020 - 7-8\%, and during the last week it dropped to $3 \%$ (21.05.2020).

In Sweden, the pandemic strategy included the following measures: 1. Do not interrupt business activities. 2. Don't disrupt people's daily lives. 3. Systematically move towards achieving collective immunity. Citizens were asked to observe social distancing precautions, but they were not required to do so and were not penalized for non-compliance. Two weeks ago, the health care system was on the verge of collapse, but the situation has improved and there has been no collapse. The number of infected people is still growing, and the death rate is higher than in other Scandinavian countries, but lower than in countries that have taken radical isolation measures. WHO now considers the Swedish model to be a possible model for countries waiting for a vaccine.

The effectiveness of COVID-19 restrictive measures to prevent the spread of infection has been studied comparatively in the states of Iowa (where they were not introduced) and Illinois, where these measures were introduced from March 21, 2020. [17]. In addition, the difference in the timing of closure of schools and businesses, population density and income levels were taken into account. The population density was higher in Iowa (114.2 people per square mile) than in Illinois (78.2 people). Summarized COVID-19 cases for 10,000 populations in both states were compared prior to the State of Illinois self-imposed isolator ordinance. After the decree, the incidence became higher in Iowa, decreased in Illinois. Within 10, 20, and 30 days of the Illinois Home Isolation Ordinance, the difference in COVID-19 cases was 0.51 per 10,000 residents (SE $0.09 ; 95 \%$, CI is from 0.69 to $0.32 ; \mathrm{p}<0.001 ;-1.15$ per 10,000 population (SE $0.49 ; 95 \%$ CI is from -2.12 to $-0.18 ; \mathrm{p}=0.02$ ) and -4.71 per 10,000 population (SE $1.99 ; 95 \% \mathrm{CI}$ is from -8.64 to $-0.78 ; \mathrm{p}=0.02$ ), respectively. These data demonstrate the effectiveness of home isolation to prevent the spread of COVID-19 even in selected regions of the country.

Medical organizations are known to be one of the frequent outbreak centers of infection. Often, when such a focal point arises in a general hospital, infected patients are transferred to other hospitals, creating a chain of infectious disease outbreaks, sometimes from infectious disease hospitals to non-infectious disease hospitals for surgical interventions. It is always a high risk of infection for patients and medical staff. It seems reasonable to introduce a surgeon to infectious diseases hospitals and to organize an operating room.

Conclusion. Analysis of the prevalence of the new coronavirus disease COVID-19 over a 4-month period from the initial "emotional" assessment based on intensive indicators is gradually moving to a system of "medical statistics". The actual epidemiological situation, trends and prognosis can be most reliably assessed and 
determined based on relative morbidity and mortality rates.

It is necessary to distinguish such indicators as "morbidity", "infection rate", "mortality" and "lethality" according to the generally accepted postulates of medical statistics and epidemiology. Proper calculation of such indicators as morbidity (number of patients with COVID-19 per 1,000/100,000 of population), the rate of infection (or reproduction), allows to objectively assess the effectiveness of various restrictive measures in dynamics, helps to make decisions about the continuation or weakening of these activities, and for the health system - about the possibilities for the near future to cope with the upcoming flow of patients or make adjustments to strengthen their resources. Approximately the same importance of another indicator - mortality (the number of deaths per 100,000 population), although it characterizes the state, the level of health care system. Indicator - lethality (\% deceased to the number of patients) - is primarily an assessment of the activities of medical organizations, medical workers, the level of medical knowledge, professionalism, the state of implementation of research results. Undoubtedly, the level of lethality is also connected with material and technical, medical equipment of medical organizations, opportunities of conversion of medical organizations into infectious diseases hospitals, organization of intensive care and resuscitation departments, providing the latter with equipment for non-invasive and invasive ventilation of the lungs, hemodialysis, efferent methods of treatment.

\section{REFERENCES}

1. Gerasimov A.N. Medical statistics. M.: MLLC "Medical News Agency" 2007; 480 (In Russ.)

2. The economic impact of coronavirus: analysis from experts at Imperial I Imperial News [cites 2020 Apr6]. Available from. https://www.imperial.ac.uk/news/196514/theeconomic-impact-coronovirusanalysis-from/

3. Plastics Trade Body Publishes First Study of Coronovirus Impact on UK Manufacturing [Internet]. [cited 2020 Mar 20] Available from:https://www.lpf.co.uk/article/plastics-trade-body-publishes-firststudy-of-coronovirus-impact-1602.aspx

4. COVID-19 is Coming for the Chemical Industry in 2020, BASF Frets I Industry Week [Internet]. [cited 2020 Mar 20] Available from:https://www.industryweek.com/supplychain/planningforecasting/article/21125045/covid19-is-coming-for-the-chemical-industry-in-2020-basf-frets.

5. Loeb $\mathrm{AB}$ Avi. Flattening the COVID-19 Curves [Internet] Scintific American Blog Networkhttps://blogs.scientificamerican.com/observations/flattening-the-covid-19-curves/

6. Knieps S. Will COVID-19 turn Germany's export-oriented economy into a weekness? [Internet]www.euractiv.com/2020. [cited 2020 Mar 23] Available from:https://www.euractiv.com/section/economy-jobs/news/will-covid-19-turn-germanys-exportoriented-economy-into-a-weakness/

7. Cumming M.J., Baldwin M.R., Abrams D. et al. Epidemiology, clinical course, and outcomes of critically ill adults with COVID-19 in New York City: a prospective cohort study.www.thelancet.com Published online. May 19, 2020.http://doi.org/10.1016/S0140-6736(20)31189-2

8. INCARC report on COVID-19 in critical are 22 May 2020. www.icnarc.org.

9. Jeon S., Ko M., Lee J. et al. Identification of antiviral drug candidates against SARS-CoV-2 from FDAapproved drugs. Antimicrobal Agents and Chemotherapy AAC Accepted Manuscript Posted Online 4 May 2020 doi: 10.1128/AAC.00819-20

10. Mehra M.R., Desai S.S., Ruschitzka F., Patel A.N. Hydroxychloroquine or chloroquine with or without a macrolide for treatment of COVID-19: a multinational registry analysis. The Lancet 2020. May 22. DOI:https://doi.org/10.1016/S0140-6736(20)31180-6

11. Morens D.M., Taubenberger J.K., Fauci A.S. Predominant role of bacterial pneumonia as a cause of death in pandemic influenza: implications for pandemic influenza preparedness. J Infect Dis. 2008. 198: 962-970

12. Macintyre C.R., Chughtai A.A., Barnes M. et al. The role of pneumonia and secondary bacterial infection in fatal and serious outcomes of pandemic influenza a (H1N1) pdm09. BMC Infect Dis 2018; 18: 637

13. Zhou F., Yu T., Du R. et al. Clinical course and risk factors for mortality of adult inpatients with 
COVID-19 in Wuhan, China: a retrospective cohort study. Lancet 2020; 395: 1054-1062

14. Chan N., Zhou M., Dong X. et al. Epidemiological and clinical characteristics of 99 cases of 2019 novel coronovirus pneumonia in Wuhan, China: a descriptive study. Lancet 2020; 395: 507-513

15. Kermack W.O., Mc Kendrick A.G., A contribution to the mathematical theory of epidemics. Proceeding of the Royal Society of London. Series A. Containing papers of a mathematical and physical character. 1927: 115(772):700-721

16. Balestrino A., Fiaschi P., Melloni I. et al in Reply TheCoronovirus Disease 2019 Global Pandemic: A Neurosurgical Treatment Algorithm. Neurosurgery.published.online: April 2020 doi: /10/1093/neuros /nyaa177/5831779.

17. Lyu W., Wehby G.L. Comparison of Estimated Rates of Coronovirus Disease 2019 (COVID-19) in Border Counties in Iowa Without a Stay-at-Home Order and Border Countries in Illinois with a Stayat-Home Order. JAMA Network Open 2020; 3(5): e2011102. Doi: 10.1001/jamanetworkopen.2020.11102

18. Tamm M.V., Coronovirus infection in Moscow: forecasts and pharmacoepidemiology. 2020; 13(1):43-51 Doi:10.17749/2070-4909.2020.13.1.43-51 (In Russ.)

19. Shen Ch., Wang Z., Zhao F. et al. Treatment of 5 Critically ill Patients with COVID-19 with Convulescent Plasma. JAMA, 2020; 323(16):1582-1589. Doi: 10.1001/jama.2020.4783

20. Duan K., Liu B., Li C. et al. Effectiveness of convalescent Plasma for COVID-19 patients in Wuhan, China. J Medical Virology 15 April 2020.http://doi.org/10.1002/jmv.25882

21. Beigel J.H., Aga E., Elie-Turenne M-C. et al. Anti-influenza immune plasma for the treatment of patients with severe influenza A: a randomized, double-Glind, phase 3 trial. The Lancet 2019: 7(11): 941-950.https://doi.org/10.1016/S2213-2600(19)30199-7 